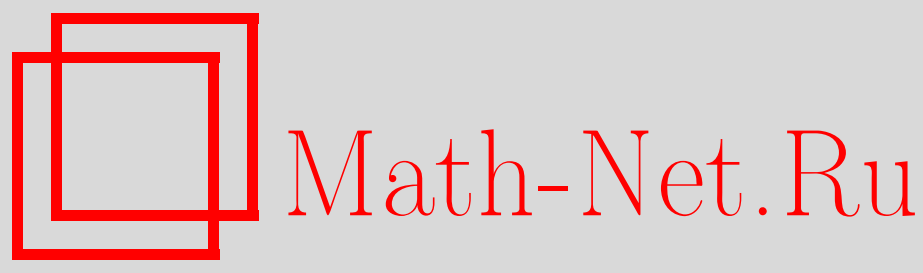

С. Б. Лебле, Преобразование Дарбу интегрируемых потенциалов в ассоциативных кольцах и квантовые и классические задачи, ТМФ, 2018, том 197, номер 1, 108-123

DOI: https://doi.org/10.4213/tmf9475

Использование Общероссийского математического портала Math-Net.Ru подразумевает, что вы прочитали и согласны с пользовательским соглашением http://www . mathnet.ru/rus/agreement

Параметры загрузки:

IP : 54.92 .164 .108

26 апреля 2023 г., 14:02:27

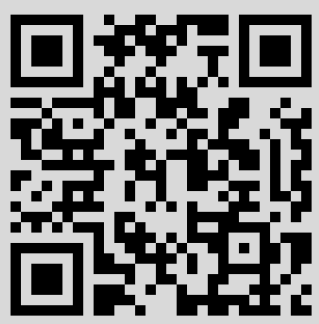




\title{
ПРЕОБРАЗОВАНИЕ ДАРБУ ИНТЕГРИРУЕМЫХ ПОТЕНЦИАЛОВ В АССОЦИАТИВНЫХ КОЛЬЦАХ И КВАНТОВЫЕ И КЛАССИЧЕСКИЕ ЗАДАЧИ
}

\begin{abstract}
Изучается задача в ассоциативных кольцах о левой и правой факторизации полиномиального дифференциального оператора, который рассматривается как оператор эволюции. В прямой сумме колец полином, возникающий в задаче деления оператора на оператор для двух коммутирующих операторов, приводит к зависящему от времени левому/правому преобразованию Дарбу, основанному на сплетающем соотношении и либо на отображениях Миуры, либо на обобщенных уравнениях Бюргерса. Сплетающие соотношения приводят к дифференциальному уравнению, включающему слабые производные. В свете применений к операторным задачам квантовой и классической механики напрямую получены квазидетерминанты или формулы для одевающих цепочек. Рассмотрено преобразование операторов рождения и уничтожения в указанных матричных кольцах. Изучается пример модели Дикке.
\end{abstract}

Ключевые слова: факторизация полиномиального дифференциального оператора, преобразование Дарбу, преобразование Дарбу-Матвеева, обобщенные преобразования Миуры, уравнения Бюргерса, цепочки в кольцах, одевание гамильтониана Дикке.

DOI: https://doi.org/10.4213/tmf9475

\section{1. ВВЕДЕНИЕ. РАЗВИТИЕ ТЕОРИИ}

Теория одевания потенциала для квантовых или классических гамильтонианов, сформулированная в терминах скобок Пуассона присоединенных операторов, содержит некоммутативные составляющие и требует обобщения. Развитие методов одевания и методов левого и правого деления оператора на оператор приводит к соответствующим соотношениям Миуры и в случае эволюционных уравнений дает некоммутативные нелинейные уравнения линеаризации типа уравнения Бюргерса.

В работе [1] были введены зависящие от времени квантово-механические потенциалы, конечно-разностные уравнения и преобразования Дарбу (ПД) для эволюционных уравнений.

*Балтийский федеральный университет им. Иммануила Канта, Калиниград, Россия. E-mail: lebleu@mail.ru 
Обобщенные бинарные полиномы Белла вводятся как удобный аппарат для получения явных выражений для преобразований. Обобщенное уравнение Бюргерса при этом оказывается линеаризуемым. Его стационарный вариант отождествляется с (обобщенным) соотношением Миуры [2]. По сравнению с книгой [2] в настоящей работе мы различаем проблемы левого и правого деления на оператор, перенося открывающиеся при этом возможности на левые и правые ПД. Все компоненты теории ПД, особенно обобщения преобразования Миуры, являются инструментами для построения квазидетерминантов и уравнений цепочки посредством ПД.

Среди применений теории, которые основаны на специальном дифференцировании, можно отметить коммутатор или скобку Пуассона как присоединенные операторы в алгебре Ли [3], [4].

Многочисленные модельные гамильтонианы в квантовой механике, выраженные в терминах операторов рождения-уничтожения, можно переписать в терминах дифференциальных операторов [5]. Среди этих моделей наиболее привлекательными являются точно решаемые случаи [6].

После введения в разделе 2 основных определений дифференциального кольца и неабелевых многочленов, которые используются при работе с дифференциальными операторам, в разделе 3 мы переходим к двум возможным правой и левой факторизациям полиномиальных дифференциальных операторов и на основе этого получаем соответствующие сплетающие отношения и ПД. Раздел 4 содержит два представления для итерационного ПД: одно задает волновые функции и потенциалы в терминах квазидетерминантов, второе - в терминах так называемых суперпотенциалов, решений правой или левой одевающих цепочек; это обобщает результаты из трудов [5], [2]. Раздел 5 посвящен одеванию точно решаемых моделей (см., например, статью [7]).

\section{2. ОБЩИЕ ЗАМЕЧАНИЯ}

2.1. Основные определения. Пусть $K$ - дифференциальное кольцо с нулевой характеристикой, с двумя дифференцированиями $D_{1}, D_{2}$, с единицей $e$ (т. е. унитарное кольцо) и с инволюцией, которая обозначается верхним индексом *. Дифференцирования и инволюция согласованы с операциями кольца $K$ : для всех $a, b \in K$

1) $\left(a^{*}\right)^{*}=a,(a+b)^{*}=a^{*}+b^{*},(a b)^{*}=b^{*} a^{*}$;

2) $D_{i}(a+b)=D_{i} a+D_{i} b, D_{i}(a b)=\left(D_{i} a\right) b+a D_{i} b$;

3) $\left(D_{i} a\right)^{*}=-D_{i} a^{*}$.

Образуем прямую сумму $K \oplus K$ двух копий кольца $K$ и зададим дифференцирование в ней как $D=D_{1} \oplus D_{2}$, предполагая, что:

4 ) операторы $D^{n}$ с различными $n$ образуют базис в $K$-модуле $\operatorname{Diff}(K)$ дифференциальных операторов; подкольцо постоянных функций есть $K_{0}$, а мультипликативная группа элементов кольца $K$ есть $G$;

$5)$ для каждого $s \in K$ существует элемент $\varphi \in K$, такой что $D \varphi=s \varphi$, это также означает, что в силу свойств инволюции существует решение уравнения $D \phi=-\phi s$.

Матричные дифференциальные кольца применяются в теории Лакса интегрируемых нелинейных уравнений, а также в классических и квантовых линейных задачах. Матрицы параметризуются переменной $x$, при этом $D$ может быть производной по этой переменной или комбинацией частных производных, удовлетворяя при этом 
условиям 1 и 2. Если $D$ является стандартным дифференцированием, то инволюция * может быть эрмитовым сопряжением. В случае, когда $D$ связана с коммутатором, она действует как $D a=[d, a]$ и $(D a)^{*}=-\left[d^{*}, a\right]$.

2.2. Вспомогательные соотношения. Левые и правые неабелевы полиномы Белла $B_{n}(s)$ задаются следующими рекуррентными соотношениями: для левых полиномов Белла

$$
B_{n}(s)=D B_{n-1}(s)+B_{n-1}(s) s,
$$

для правых полиномов Белла

$$
B_{n}^{+}(s)=-D B_{n-1}^{+}(s)+s B_{n-1}^{+}(s), \quad n=1,2, \ldots,
$$

при этом имеет место "начальное условие" $B_{0}(s)=e$. Отсюда

$$
B_{1}(s)=s, \quad B_{2}(s)=D s+s^{2}, \ldots .
$$

Пусть

$$
L=\sum_{n=0}^{N} a_{n} D^{n}, \quad a_{n} \in K,
$$

есть дифференциальный оператор порядка $N$. Мы изучаем правое и левое деление оператора $L$ на оператор $L_{s}=D-s$. Предположим, что

$$
L=M L_{s}+r, \quad L=L_{s} M^{+}+r^{+},
$$

где $M$ и $M^{+}$- результаты правого и левого деления, а $r$ и $r^{+}$- остатки от деления. Если имеет место представление (5), то остаток $r$ и результат деления $M$ записываются как

$$
r=\sum_{n=0}^{N} a_{n} B_{n}(s), \quad M=\sum_{n=0}^{N-1} b_{n} D^{n},
$$

где коэффициенты $b_{n}$ могут быть выражены через двухиндексные полиномы (см. раздел 5):

$$
b_{n}=\sum_{k=n+1}^{N} a_{k} B_{k-1, n}(s), \quad n=0,1, \ldots N-1 .
$$

Как следствие мы получаем, что, для того чтобы линейный оператор $L$ делился справа на $L_{s}$ без остатка, необходимо и достаточно, чтобы $s$ являлось решением дифференциального уравнения

$$
\sum_{n=0}^{N} a_{n} B_{n}(s)=0
$$

Если это уравнение выполнено, то оператор $L$ представляется в виде произведения $L=M L_{s}$, где оператор $M$ задан формулами (6) и (7).

Уравнение (8) нелинейно. При $N=2$ это уравнение типа Риккати, известное в теории уравнения КдФ как отображение Миуры. Таким образом, естественно назвать (8) обощенным правым уравнением Миуры. Оно связывает функцию $s$ с коэффициентами оператора $L$. Левое уравнение Миуры получается путем аналогичного обобщения на основе факторизации, заданной в (5). 
Пусть обратимая функция $\varphi$ является решением линейного дифференциального уравнения

$$
\sum_{n=0}^{N} a_{n} D^{n} \varphi=0 .
$$

Тогда оператор $L$, заданный в (4), делится справа на $L_{s}$, где $s=\varphi^{\prime} \varphi^{-1}$ и $\varphi^{\prime} \equiv D \varphi$. Таким образом, $s$ - решение правого уравнения Миуры (8).

Для решения задачи левого деления запишем результат деления в виде

$$
M^{+}=\sum_{n=0}^{N-1} b_{n}^{+} D^{n} .
$$

Теперь нам следует определить коэффициенты $b_{n}^{+}, n=0,1, \ldots, N-1$. Подставим выражение (10) в правую часть второго уравнения в (5) и приравняем коэффициенты при операторах $D^{n}$, получим

$$
\begin{gathered}
b_{N-1}^{+}=a_{N}, \quad b_{n}^{+}=a_{n+1}-L_{s} b_{n+1}^{+}, \quad n=0,1, \ldots, N-2, \\
r^{+}=a_{0}-L_{s} b_{0}^{+} .
\end{gathered}
$$

Формулы (5), (11), (12) дают решение задачи левого деления оператора $L$ на $L_{s}$ (детали см. в книге [2]).

Нелинейное уравнение $r^{+}=0$ называется обобщенным левым уравнением Миуры. Его также можно линеаризовать. Предположим, что обратимый элемент $\varphi$ удовлетворяет линейному дифференциальному уравнению

$$
\sum_{n=0}^{N}(-1)^{n} B_{n}(s)^{+} a_{n} D^{n} \varphi=0 .
$$

Тогда оператор $L$, заданный равенством (4), делится слева на оператор $L_{s}$, в то время как оператор (12) линеаризуется с помощью "антиподстановки Коула-Хопфа" $s=-\varphi^{-1} \varphi^{\prime}$. Функция $s$ является решением левого уравнения Миуры (13).

\section{3. ПД ПОСРЕДСТВОМ ФАКТОРИЗАЦИИ}

3.1. Правое уравнение Миуры. Правое ПД. Правое уравнение Миуры

$$
\sum_{k=0}^{N} B_{k}(s) a_{k}=0
$$

дает

$$
L=M L_{s},
$$

и это соотношение можно напрямую применить к задаче на собственные значения $L \psi=M L_{s} \psi=\psi \lambda$, где $\lambda \in K$ есть "спектральный параметр". Это приводит к уравнению $L_{s} M L_{s} \psi=L_{s} \psi \lambda$, которое можно переписать как $L_{s} M \tilde{\psi}=\tilde{L} \tilde{\psi} \lambda$, где равенства $\tilde{\psi}=L_{s} \psi$ и $\tilde{L}=L_{s} M$ задают правое ПД.

Если $s=(D \phi) \phi^{-1}, L_{s}=\phi D \phi^{-1}$, то формально $L_{s}^{-1}=\phi D^{-1} \phi^{-1}$. 
3.2. Левое уравнение Миуры и левое ПД. Левое уравнение Миуры

$$
r^{+}=\sum_{k=0}^{N}(-1)^{k} L_{s}^{k} a_{k}=0
$$

которое эквивалентно уравнению (14), теперь записывающемуся как

$$
r^{+}=\sum_{k=0}^{N} B_{k}^{+}(s) a_{k}=0
$$

дает $L=L_{s^{+}} M^{+}$, где

$$
L_{s^{+}}=D-s^{+},
$$

но в “левом" случае $s^{+}=-\phi^{-1} D(\phi)$ подразумевается, что $\phi^{-1}$ существует.

Следовательно, задачу на собственные значения можно факторизовать следующим образом: $L \psi=L_{s^{+}} M^{+} \psi=\psi \lambda$. Подставляя $\psi=L_{s^{+}} \phi$, мы имеем

$$
L_{s^{+}} M^{+} L_{s^{+}} \phi=L_{s^{+}} \phi \lambda \text {. }
$$

Пусть существует $\phi^{-1}$, тогда действие оператора $L_{s^{+}}^{-1}=\left(\phi^{-1} D \phi\right)^{-1}=\phi^{-1} D^{-1} \phi$ дает уравнение $M^{+} L_{s^{+}} \phi=L^{+} \phi=\phi \lambda$. Положим $L^{+}=M^{+} L_{s^{+}}$, тем самым левое ПД задается как $\phi=L_{s^{+}}^{-1} \psi$. Сравнивая этот результат с обычной формой ПД $\tilde{L}=L_{s} M$, можно использовать те же обозначения для левых полиномов Белла, что в (2), или напрямую использовать выражение

$$
L^{+}=M^{+} L_{s^{+}}=\sum_{n=0}^{N-1} b_{n}^{+} D^{n} L_{s^{+}}
$$

Выделяя коэффициенты, получаем на основе (11) ПД для коэффициентов полиномов; для $N=2$ это подробно показано в следующем пункте.

3.3. Случай $N=2$. В этом случае левое соотношение Миуры имеет вид $r^{+}=\sum_{k=0}^{2} B_{k}^{+}(s) a_{k}=B_{0}^{+}(s) a_{0}+B_{1}^{+}(s) a_{1}+B_{2}^{+}(s) a_{2}=a_{0}+s^{+} a_{1}-\left(D s^{+}\right) a_{2}+\left(s^{+}\right)^{2} a_{2}=0$, при этом $b_{1}^{+}=a_{2}, b_{0}^{+}=a_{1}-L_{s}+b_{1}^{+}$. Напомним, что $r^{+}=a_{0}-L_{s}^{+} b_{0}^{+}=0$ и

$$
\begin{aligned}
L^{+} & =M^{+} L_{s^{+}}=\left(b_{0}^{+}+b_{1}^{+} D\right) L_{s^{+}}=\left(a_{1}-\left(L_{s^{+}} a_{2}\right)+a_{2} D\right) L_{s^{+}}= \\
& =\left(a_{1}+a_{2} D-\left(D a_{2}\right)+s^{+} a_{2}\right)\left(D-s^{+}\right)= \\
& =\left(a_{1}+a_{2} D-\left(D a_{2}\right)+s^{+} a_{2}\right) D-\left(a_{1}+a_{2} D-\left(D a_{2}\right)+s^{+} a_{2}\right) s^{+}= \\
& =a_{1} D+a_{2} D^{2}-\left(D a_{2}\right) D+s^{+} a_{2} D-a_{1} s^{+}-a_{2} D s^{+}+\left(D a_{2}\right) s^{+}-s^{+} a_{2} s^{+} .
\end{aligned}
$$

Поэтому

$$
\begin{aligned}
& a_{2}^{+}=a_{2}, \quad a_{1}^{+}=a_{1}-\left(D a_{2}\right)+s^{+} a_{2}-a_{2} s^{+}, \\
& a_{0}^{+}=-a_{1} s^{+}-a_{2}\left(D s^{+}\right)+\left(D a_{2}\right) s^{+}-s^{+} a_{2} s^{+} .
\end{aligned}
$$

Добавляя нулевое слагаемое

$$
r^{+}=a_{0}+s^{+} a_{1}-\left(D s^{+}\right) a_{2}+\left(s^{+}\right)^{2} a_{2}=0,
$$


приходим к соотношению

$$
a_{0}^{+}=a_{0}+\left[s^{+}, a_{1}\right]-a_{2}\left(D s^{+}\right)+\left(D a_{2}\right) s^{+}-s^{+} a_{2} s^{+}-\left(D s^{+}\right) a_{2}+\left(s^{+}\right)^{2} a_{2} .
$$

Если $a_{2}=-1$, то

$$
\begin{gathered}
a_{1}^{+}=a_{1}, \quad a_{0}^{+}=-a_{1} s^{+}+\left(D s^{+}\right)+s^{+} s^{+} \\
r^{+}=a_{0}+s^{+} a_{1}+\left(D s^{+}\right)-\left(s^{+}\right)^{2}=0 .
\end{gathered}
$$

Комбинируя эти соотношения, получаем

$$
a_{1}^{+}=a_{1}, \quad a_{0}^{+}=a_{0}+2\left(D s^{+}\right)+\left[s^{+} a_{1}\right] .
$$

Правое деление приводит к уравнениям

$$
\tilde{L}=L_{s} M, \quad M=b_{0}+b_{1} D,
$$

где

$$
\begin{gathered}
b_{0}=\sum_{k=1}^{2} a_{k} B_{k-1,0}(s)=a_{1} B_{0,0}(s)+a_{2} B_{1,0}(s)=a_{1}+a_{2}, \quad b_{1}=a_{2} B_{1,1}(s)=a_{2} s \\
M=a_{1}+a_{2}+a_{2} s D=\sum_{n=1}^{N} a_{n} H_{n-1}=\sum_{n=0}^{N-1} b_{n} \\
\tilde{L}=L_{s} M=(D-s)\left(a_{1}+a_{2}+a_{2} s D\right)
\end{gathered}
$$

Для коэффициентов в случае $N=2$ имеем

$$
\begin{gathered}
\tilde{a}_{2}=a_{2}, \quad \tilde{a}_{1}=a_{1}+D a_{2}+\left[a_{2}, s\right], \\
\tilde{a}_{0}=a_{0}+\left[a_{1}, s\right]+\left(D a_{1}\right)+\left[a_{2}, s\right] s+2 a_{2} D s+\left(D a_{2}\right) .
\end{gathered}
$$

При $a_{2}=-1$ преобразования упрощаются и задаются как

$$
\tilde{a}_{1}=a_{1}, \quad \tilde{a}_{0}=a_{0}+D a_{1}+\left[a_{1}, s\right]-2(D s) .
$$

Для левого преобразования имеем (25).

Напомним, линеаризация проведена с помощью “антиподстановки" Коула-Хопфа $s^{+}=-\phi^{-1} \phi^{\prime}$. Сравним с правым преобразованием Миуры (14) в случае $N=2$ :

$$
a_{2}\left(s^{\prime}+s^{2}\right)+a_{1} s+a_{0}=0 .
$$

В случае уравнения Кд $\Phi$, когда $a_{2}=-1, a_{1}=0$, взяв $a_{0}=u(x, t)-\mu$, получаем правое соотношение Миуры $-\left(s^{\prime}+s^{2}\right)+u=\mu$. Левое соотношение при этом имеет вид $\left(s^{+}\right)^{\prime}-\left(s^{+}\right)^{2}+u=\mu$ и отличается только знаком при $s^{\prime}$. Таким образом, уравнения кажутся эквивалентными. Иная ситуация складывается в неабелевом случае.

\section{4. ИТЕРАЦИИ. КВАЗИДЕТЕРМИНАНТЫ И ЦЕПОЧКИ}

4.1. Квазидетерминанты. Для дифференцируемого элемента $f$ кольца $R$ положим $\mathcal{D}(\phi ; f)=f^{\prime}-\phi^{\prime} \phi^{-1} f$, где $\phi, f$ - линейно независимые собственные функции спектральной задачи для оператора (4). Следуя работе [8], назовем $\mathcal{D}(\phi ; f)$ ПД 
функции $f$, задающимся функцией $\phi$. Данное определение позволяет повторить преобразование с другой линейно независимой функцией $\phi_{1}$, и в результате мы имеем $\mathcal{D}\left(\phi_{1} ; \mathcal{D}(\phi ; f)\right)=\mathcal{D}(\phi ; f)^{\prime}-\phi_{1}^{\prime} \phi_{1}^{-1} \mathcal{D}(\phi ; f)$.

Пусть $\phi_{1}, \ldots, \phi_{k} \in R$. Определим по индукции итерационное ПД $\mathcal{D}\left(\phi_{k}, \ldots, \phi_{1} ; f\right)$ следующим образом. Для $k=1$ оно совпадает с ПД, заданным выше. Пусть теперь $k>1$. Преобразование $\mathcal{D}\left(\phi_{k}, \ldots, \phi_{1} ; f\right)$ определено, если известны преобразования $\mathcal{D}\left(\phi_{k}, \ldots, \phi_{2} ; f\right)$ и $\mathcal{D}\left(\phi_{k} ; f\right)$, причем $\mathcal{D}\left(\phi_{k} ; f\right)$ обратимо. В этом случае

$$
\mathcal{D}\left(\phi_{k}, \ldots \phi_{1} ; f\right)=\mathcal{D}\left(\mathcal{D}\left(\phi_{k}, \ldots, \phi_{2} ; f\right) ; \mathcal{D}\left(\phi_{1} ; f\right)\right) .
$$

Если обратимы все квадратные подматрицы $\left(\phi_{i}^{(j)}\right), i=1, \ldots, k, j=k-1, \ldots, 0$, то суперматрица Вандермонда определяет квазидетерминант

$$
\widehat{\mathcal{D}}\left(\phi_{k}, \ldots, \phi_{1} ; f\right)=\left|\begin{array}{cccc}
f^{(k)} & \phi_{1}^{(k)} & \ldots & \phi_{k}^{(k)} \\
\vdots & \vdots & \ddots & \vdots \\
f & \phi_{1} & \ldots & \phi_{k}
\end{array}\right|
$$

(мы используем шляпку для обозначения квазидетерминанта). Итерационное ПД задается двумя квазидерминантами [2].

4.2. Цепочки левых и правых ПД. Чтобы пояснить алгоритм, ограничимся случаем $N=2$. Пусть $a_{0}=u$ есть базовое поле. Выразим $u$ из левого соотношения Миуры как

$$
u=a_{0}=-s^{+} a_{1}-\left(D s^{+}\right) a_{2}-\left(s^{+}\right)^{2} a_{2}
$$

или, аналогично, из правого соотношения как

$$
u=a_{0}=-a_{2}\left(s^{\prime}+s^{2}\right)-a_{1} s .
$$

Изучая итерации левого и правого ПД, мы приходим к двум типам цепочек ПД. Оба отображения (32), (33) позволяют для любого $n$ выразить $u$ через функции $s$ или $s^{+}$, называемые суперпотенциалами:

$$
u_{n}=-s_{n}^{+} a_{1}-\left(D s_{n}^{+}\right) a_{2}-\left(s_{n}^{+}\right)^{2} a_{2}
$$

или, для правого преобразования,

$$
u_{n}=-a_{2}\left(s_{n}^{\prime}+s_{n}^{2}\right)-a_{1} s_{n}
$$

Рассматривая формулы (29), мы видим, что условие инвариантности формы оператора $L$ выполнено, если выбрать коэффициент $a_{2}$ так, что для правого ПД мы имеем $D a_{2}+\left[a_{2}, s\right]=0$, а для левого имеем $-\left(D a_{2}\right)+s^{+}\left[a_{2}, s^{+}\right]=0$, при этом результаты упрощаются следующим образом. Левые ПД в терминах потенциалов записываются как $u_{n+1}^{+}=u_{n}+2\left(D s_{n}^{+}\right)+\left[s_{n}^{+}, a_{1}\right]$. В случае правых преобразований при условии $D a_{2}=\left[a_{2}, s\right]=0$ мы получаем

$$
u_{n+1}=u_{n}+\left[a_{1}, s_{n}\right]+\left(D a_{1}\right)+2 a_{2} D s_{n}
$$

и записываем уравнения цепочки ПД для суперпотенциалов как

$$
-a_{2}\left(D s_{n+1}+s_{n+1}^{2}\right)-a_{1} s_{n+1}=a_{2} D s_{n}-a_{2} s_{n}^{2}-a_{1} s_{n}+\left[a_{1}, s_{n}\right]+D a_{1} \text {. }
$$


Случай левых преобразований при аналогичных условиях приводит к уравнениям

$$
u_{n+1}^{+}=u_{n}-a_{1} s_{n}^{+}-a_{2}\left(D s_{n}^{+}\right)+\left(D a_{2}\right) s_{n}^{+}-s_{n}^{+} a_{2} s_{n}^{+} .
$$

Далее, левое ПД в терминах “суперпотенциалов" имеет вид

$$
\begin{aligned}
-s_{n+1}^{+} a_{1}- & \left(D s_{n+1}^{+}\right) a_{2}-\left(s_{n+1}^{+}\right)^{2} a_{2}= \\
& =-s_{n}^{+} a_{1}-\left(D s_{n}^{+}\right) a_{2}-\left(s_{n}^{+}\right)^{2} a_{2}+\left[a_{1}, s_{n}^{+}\right]+\left(D a_{1}\right)+2 a_{2} D s_{n}^{+} .
\end{aligned}
$$

Оба соотношения (37) и (39) мы называем общими уравнениями цепочки ПД.

Полученные результаты естественным образом обобщаются на все случаи, когда выполнены основные свойства ПД: преобразование, получающееся на $n$-й итерации, есть линейная функция от $T^{n} \psi$, и оператор преобразования имеет $n$-мерное ядро [2].

\section{5. ПД И ЭВОЛЮЦИЯ. ОБОБЩЕННЫЕ УРАВНЕНИЯ БЮРГЕРСА}

Проблема деления оператора на оператор напрямую связана с ПД. Чтобы это пояснить, предположим, что в кольце $K$ существует еще один оператор дифференцирования $D_{0}$, коммутирующий с $D$. Это может быть диффреренцирование по параметру $t$.

Введем вспомогательное коммутационное соотношение

$$
L_{s} r=r L_{s}+D r+[r, s]
$$

В самом деле,

$$
\begin{aligned}
L_{s} r-r L_{s} & =(D-s) r-r(D-s)=D r-s r-r D+r s= \\
& =r D+D r-s r-r D+r s=D r+[r, s] .
\end{aligned}
$$

Принимая во внимание равенства (40) и (15), мы приходим к соотношению

$$
L_{s}\left(D_{0}-L\right)=\left(D_{0}-\tilde{L}\right) L_{s}+D_{0} s-D r-[r, s],
$$

где

$$
\tilde{L}=L_{s} M+r
$$

В результате получаем важный вывод: если функция $s$ удовлетворяет уравнению

$$
D_{0} s=D r+[r, s]
$$

то оператор $L_{s}$ является сплетающим для операторов $D_{0}-L$ и $D_{0}-\tilde{L}$ :

$$
L_{s}\left(D_{0}-L\right)=\left(D_{0}-\tilde{L}\right) L_{s}
$$

Явное выражение для $\tilde{L}$ можно получить в терминах операторов (42) и (15).

ЗАмечАниЕ. Факторизация нам не требуется $(r=0)$, элемент $s$ должен только быть решением "обобщенного уравнения Бюргерса" (43). А именно, запишем уравнение (43) в явном виде, используя выражение (15). Мы утверждаем, что, для 
того чтобы выполнялось сплетающее соотношение (44), необходимо и достаточно, чтобы $s$ являлось решением уравнения

$$
D_{0} s=\sum_{n=0}^{N}\left(D a_{n} B_{n}(s)+a_{n} B_{n+1}(s)-s a_{n} B_{n}(s)\right) .
$$

Используя соотношение (1), мы получаем еще одно представление, удобное для анализа:

$$
D_{0} s=\sum_{n=0}^{N}\left(D\left(a_{n} B_{n}(s)\right)+\left[a_{n}, s\right] B_{n}(s) s\right) .
$$

Уравнение (45) нелинейно, но линеаризуемо. Предложенная нами формула (43) наиболее компактна и удобна для дальнейшего исследования, например, в рамках техники билинеаризации Хироты.

В случае, когда коэффициенты коммутируют и $L=D^{2}$, уравнение (45) приобретает вид известного уравнения Бюргерса. По этой причине и вследствие возможности проинтегрировать уравнение (45) с помощью преобразования Коула-Хопфа естественно назвать (45) обобщенным уравнением Бюргерса. Предположим, что обратимая функция $\varphi$ является решением линейного дифференциального уравнения $D_{0} \varphi=L \varphi$. Тогда функция $s$ удовлетворяет обобщенному уравнению Бюргерса (45).

Пусть функции $\psi$ и $\varphi$ являются решениями уравнений $D_{0} \psi=L \psi$ и $D_{0} \varphi=L \varphi$, при этом функция $\varphi$ обратима. Тогла функции $\tilde{\psi}=L_{s} \psi=D \psi-s \psi$ и $s=(D \varphi) \varphi^{-1}$ являются решениями уравнения $D_{0} \tilde{\psi}=\tilde{L} \tilde{\psi}$. Последнее утверждение завершает доказательство теоремы Матвеева для дифференциальных многочленов (см., например, работу [1]) в ее неабелевом варианте.

Равенство (42) дает представление преобразованных операторов в терминах обобщенных полиномов Белла. Явные выражения для коэффициентов после преобразования имеют вид

$$
\begin{gathered}
\tilde{a}_{N}=a_{N}, \\
\tilde{a}_{k}=a_{k}+\sum_{n=k+1}^{N}\left(a_{n} B_{n, n-k}+\left(a_{n}^{\prime}-s a_{n}\right) B_{n-1, n-1-k}\right), \quad k=0, \ldots, N-1 .
\end{gathered}
$$

Функции $B_{m, n}$ были введены в работе [9]. Воспроизведем здесь определения и некоторые утверждения об этих функциях.

ОПРЕДЕЛЕНИЕ. Уравнения $B_{n, 0}(\sigma)=1, n=0,1,2, \ldots$, и рекуррентные соотношения

$$
\begin{array}{ll}
B_{n, k}(\sigma)=B_{n-1, k}(\sigma)+D B_{n-1, k-1}(\sigma), & k=1, \ldots, n-1, \quad n=2,3, \ldots, \\
B_{n, n}(\sigma)=D B_{n-1, n-1}(\sigma)+B_{n}(\sigma), & n=1,2, \ldots,
\end{array}
$$

определяют обобщенные полиномы Белла $B_{m, n}$.

Можно получить следующие формулы:

$$
B_{n, n-k+1}(\sigma)=\sum_{i=k}^{n}\left(\begin{array}{l}
i \\
k
\end{array}\right) B_{n, n-i}(\sigma) D^{i-k} \sigma, \quad k=1, \ldots, n, \quad n=0,1,2, \ldots,
$$




$$
B_{n+1}(\sigma)=\sum_{i=0}^{n} B_{n, n-i}(\sigma) D^{i} \sigma, \quad n=0,1,2, \ldots
$$

Они связывают неабелевы и обощенные полиномы Белла:

$$
B_{n+1}(\sigma)=\sum_{i=0}^{n} B_{n, i}(v) D^{n-i} \sigma, \quad n=0,1,2, \ldots
$$

Расчет первых трех обобщенных полиномов Белла непосредственно из определения дает

$$
\begin{gathered}
B_{n, 1}(\sigma)=\sigma, \quad B_{n, 2}(\sigma)=\sigma^{2}+n D \sigma \\
B_{n, 3}(\sigma)=\sigma^{3}+n \sigma^{\prime} \sigma+(n-1) \sigma D \sigma+\left(\begin{array}{l}
n \\
2
\end{array}\right) D^{2} \sigma .
\end{gathered}
$$

\section{6. ЛЕВОЕ ПД И ЭВОЛЮЦИЯ}

Введем вспомогательное коммутационное соотношение

$$
L_{s} r^{+}=r^{+} L_{s}+D r^{+}+\left[r^{+}, s\right] .
$$

В самом деле,

$$
\begin{aligned}
L_{s} r^{+}-r^{+} L_{s} & =(D-s) r^{+}-r^{+}(D-s)=D r^{+}-s r^{+}-r^{+} D+r^{+} s= \\
& =r^{+} D+D r^{+}-s r^{+}-r^{+} D+r^{+} s=D r^{+}+\left[r^{+}, s\right] .
\end{aligned}
$$

Принимая во внимание соотношения (48) и (18), мы приходим к уравнению

$$
L_{s}\left(D_{0}-L\right)=\left(D_{0}-\tilde{L}\right) L_{s}+D_{0} s-D r^{+}-\left[r^{+}, s\right],
$$

где $\tilde{L}=L_{s} M^{+}+r^{+}$.

Соответствующее обобщенное уравнение Бюргерса $D_{0} s-D r^{+}-\left[r^{+}, s\right]=0$ влечет сплетающее соотношение $L_{s}\left(D_{0}-L\right)=\left(D_{0}-\tilde{L}\right) L_{s}$, которое в свою очередь приводит к уравнению $D_{0} \tilde{\phi}=\left(L_{s} M^{+}+r^{+}\right) \tilde{\phi}$, если $\left(D_{0}-L\right) \psi=0$; решением этого уравнения является функция $\tilde{\psi}=L_{s} \phi$.

Временно́е неабелево уравнение Шредингера. Простейшая квантовая эволюция

$$
i \psi_{t}=H \psi=\left(-D^{2}+u(x, t)\right) \psi
$$

задается условиями $a_{2}=-1, a_{1}=0, a_{0}=u(x, t)$. Мы, однако, допускаем, что потенциал и суперпотенциал являются неабелевыми и

$$
D_{0} s=\sum_{n=0}^{2}\left(D\left(a_{n} B_{n}(s)\right)+\left[a_{n}, s\right] B_{n}(s) s\right) .
$$

Правое уравнение Бюргерса имеет вид $s_{t}=D\left(-\left(s^{\prime}+s^{2}\right)+u\right), \tilde{L}=L_{s} M+r$. Его применение к абелевому кольцу хорошо известно в теории уравнения КП. Недавние результаты для множественных волн-убийц см. в работе [10]. 
Решения приведенного выше нелинейного уравнения Шредингера образуют $2 n$ параметрическое семейство гладких рациональных решений уравнения КП-I

$$
4 u_{t}+6 u u_{x}+u_{x x x}=3 u_{y y}
$$

Заменим в уравнении $t$ на $y$ и 3 на $t$. Функция $f(k ; x ; y ; t):=e^{k x+i k^{2} y+k^{3} t+\phi(k)}$, где $\phi(k):=\Phi(k)-\varphi_{3} k^{3}$, удовлетворяет системе уравнений

$$
f_{t}=f_{x x x}, \quad i f_{y}-f_{x x}=0 .
$$

Явное решение второго уравнения записывается как

$$
u(x ; y ; t)=2 \partial_{x x} \ln W\left(f_{1} ; \ldots ; f_{2 n}\right)=2\left(|v|^{2}-B^{2}\right),
$$

где $v$ - решение нелинейного уравнения Шредингера, и также представляет интерес в одномерной квантовой механике. Связь с теорией нелинейного уравнения Шредингера была обнаружена в упомянутой статье [10]. Потенциал считается ограниченным сверху и снизу. Соответствующее решение временного уравнения Шредингера имеет вид

$$
\psi:=\frac{W\left(f_{1}, \ldots, f_{n}, f\right)}{W\left(f_{1}, \ldots, f_{n}\right)}
$$

и, следовательно, дает модель потенциала, зависящего от времени, вместе с соответствующим решением уравнения Шредингера.

\section{7. СТАЦИОНАРНЫЙ СЛУЧАЙ}

Интересен случай оператора $L$ с некоммутирующими коэффициентами, даже если взять стационарное решение уравнения эволюции. В этом случае имеем

$$
\sigma_{t}=\phi_{x t} \phi^{-1}-\phi_{x} \phi^{-1} \phi_{t} \phi^{-1}=0
$$

Следовательно, первое слагаемое в сумме (45) мы можем записать как

$$
\left.D a_{0}+a_{0} s-s a_{0}+\sum_{n=1}^{N} D\left(a_{n} B_{n}(s)\right)+\left[a_{n} s\right] B_{n}(s) s\right)=0 .
$$

Возьмем в качестве примера $a_{0}(\hat{A}), \hat{A} \in K$. Тогда функция $s$ должна также зависеть от оператора $\hat{A}$, а коэффициент $a_{0}$ коммутирует с $s$. Вообще говоря, соотношение (43) означает, что если все коэффициенты $a_{n}$ коммутируют с $s$, то мы приходим к уравнению $D r=0$; таким образом, получаем, что $r=C, D C=0$.

\section{8. ПРИМЕР. МОДЕЛЬ ДИККЕ}

8.1. Решение задачи на собственные значения. Пусть система из $N$ атомов взаимодействует с одномодовым полем излучения. Суть совместного поведения частиц заключается в том, что для атомных систем атомные диполи когерентно взаимодействуют с излучением. В последние годы модель Дикке стала вновь вызвать интерес из-за наличия в ней фазовых переходов и потому, что она представляет 
собой простую модель системы, в которой можно найти многочастичную запутанность. Простая модель двухатомной системы служит полезным введением в структуру энергетических уровней модели Дикке. В представлении Фока гамильтониан модели имеет вид

$$
H_{0}=\omega_{\mathrm{c}} a^{+} a+\omega_{0} S_{z}+g\left(a^{+} S_{-}+a S_{+}\right) .
$$

Здесь $\omega_{\mathrm{c}}$ - частота моды резонатора, $\Omega_{0}$ - резонансная частота атомов и $g$ - константа связи в атоме.

Чтобы найти собственные значения, заметим, что число возбуждений $a^{+} a+S_{z}$ коммутирует с гамильтонианом $H_{0}$, и тогда при $S=1$ мы получаем линейную комбинацию состояний Дике, соответствующих состоянию $|n\rangle$, так что число возбуждений можно считать постоянной величиной $n$ (не путать с числом фотонов). Пусть

$$
|1\rangle=\left(\begin{array}{l}
1 \\
0 \\
0
\end{array}\right), \quad|0\rangle=\left(\begin{array}{l}
0 \\
1 \\
0
\end{array}\right), \quad|-1\rangle=\left(\begin{array}{l}
0 \\
0 \\
1
\end{array}\right) .
$$

Выберем матрицы в уравнении (55) как

$$
S_{z}=\left(\begin{array}{rrr}
1 & 0 & 0 \\
0 & 0 & 0 \\
0 & 0 & -1
\end{array}\right), \quad S_{-}=\left(\begin{array}{lll}
0 & 0 & 0 \\
1 & 0 & 0 \\
0 & 1 & 0
\end{array}\right), \quad S_{+}=\left(\begin{array}{lll}
0 & 1 & 0 \\
0 & 0 & 1 \\
0 & 0 & 0
\end{array}\right) .
$$

С помощью известных соотношений $a=\left(\partial_{x}+x\right) / \sqrt{2}, a^{+}=\left(\partial_{x}-x\right) / \sqrt{2}$ получаем

$$
H_{0}=-\frac{\omega_{\mathrm{c}}}{2} \partial_{x}^{2}+\frac{g}{\sqrt{2}}\left(S_{-}+S_{+}\right) \partial_{x}+\frac{\omega_{\mathrm{c}}}{2}\left(x^{2}+1\right)+\frac{g x}{\sqrt{2}}\left(S_{-}+S_{+}\right)+\omega_{0} S_{z},
$$

следовательно,

$$
a_{1}=\frac{g}{\sqrt{2}}\left(S_{-}+S_{+}\right), \quad a_{0}=\frac{\omega_{\mathrm{c}}}{2}\left(x^{2}+1\right)+\frac{g x}{\sqrt{2}}\left(S_{-}+S_{+}\right)+\omega_{0} S_{z} .
$$

Напомним, что

$$
a^{+} \phi_{n}=\sqrt{n+1} \phi_{n+1}, \quad a \phi_{n}=\sqrt{n} \phi_{n-1},
$$

и положим $\phi_{n}=|n\rangle$.

В базисе $|i\rangle|n\rangle, i=0, \pm 1, n \in \mathbb{Z}$, подпространство элементов вида $A|1\rangle|n-1\rangle+$ $B|0\rangle|n\rangle+C|-1\rangle|n+1\rangle$ является инвариантным относительно действия гамильтониана. Действие гамильтониана записывается как

$$
\begin{aligned}
H_{0}|1\rangle|n-1\rangle & =\left(\omega_{\mathrm{c}} a^{+} a+\omega_{0} S_{z}+g\left(a^{+} S_{-}+a S_{+}\right)\right)|1\rangle|n-1\rangle= \\
& =\left(\omega_{\mathrm{c}}(n-1)+\omega_{0}\right)|1\rangle|n-1\rangle+g \sqrt{n}|0\rangle|n\rangle, \\
H_{0}|0\rangle|n\rangle & =\left(\omega_{\mathrm{c}} a^{+} a+\omega_{0} S_{z}+g\left(a^{+} S_{-}+a S_{+}\right)\right)|0\rangle|n\rangle= \\
& =\omega_{\mathrm{c}} n|0\rangle|n\rangle+g \sqrt{n+1}|-1\rangle|n+1\rangle+g \sqrt{n}|1\rangle|n-1\rangle, \\
H_{0}|-1\rangle|n+1\rangle & =\left(\omega_{\mathrm{c}} a^{+} a+\omega_{0} S_{z}+g\left(a^{+} S_{-}+a S_{+}\right)\right)|-1\rangle|n+1\rangle= \\
& =\left(\omega_{\mathrm{c}}(n+1)-\omega_{0}\right)|-1\rangle|n+1\rangle+g \sqrt{n+1}|0\rangle|n\rangle .
\end{aligned}
$$

Задача на собственные значения

$$
\begin{gathered}
A\left(\omega_{\mathrm{c}}(n-1)+\omega_{0}\right)+B g \sqrt{n}=\lambda A, \quad A g \sqrt{n}+B \omega_{\mathrm{c}} n+C g \sqrt{n+1}=\lambda B, \\
B g \sqrt{n+1}+C\left(\omega_{\mathrm{c}}(n+1)-\omega_{0}\right)=\lambda C
\end{gathered}
$$


имеет следующее решение:

$$
\begin{gathered}
A\left(\omega_{\mathrm{c}}(n-1)+\omega_{0}-\lambda\right)+B g \sqrt{n}=0, \quad A g \sqrt{n}+B\left(\omega_{\mathrm{c}} n-\lambda\right)+C g \sqrt{n+1}=0, \\
B=-C \frac{\omega_{\mathrm{c}}(n+1)-\omega_{0}-\lambda}{g \sqrt{n+1}} .
\end{gathered}
$$

Далее,

$$
\begin{gathered}
A\left(\omega_{\mathrm{c}}(n-1)+\omega_{0}-\lambda\right)-C \frac{\omega_{\mathrm{c}}(n+1)-\omega_{0}-\lambda}{g \sqrt{n+1}} g \sqrt{n}=0, \\
A=\frac{C}{g \sqrt{n}}\left[\left(\frac{\omega_{\mathrm{c}}(n+1)-\omega_{0}-\lambda}{g \sqrt{n+1}}\right)\left(\omega_{\mathrm{c}} n-\lambda\right)-g \sqrt{n+1}\right], \quad B=-C \frac{\omega_{\mathrm{c}}(n+1)-\omega_{0}-\lambda}{g \sqrt{n+1}}
\end{gathered}
$$

и

$$
\begin{aligned}
{\left[\left(\omega_{\mathrm{c}}(n+1)-\omega_{0}-\lambda\right)\left(\omega_{\mathrm{c}} n-\lambda\right)-g^{2}(n+1)\right] } & \left(\omega_{\mathrm{c}}(n-1)+\omega_{0}-\lambda\right)- \\
& -\left(\omega_{\mathrm{c}}(n+1)-\omega_{0}-\lambda\right) g^{2} n=0 .
\end{aligned}
$$

В точке резонанса $\omega_{\mathrm{c}}=\omega_{0}$ мы получаем уравнение

$$
\left(\omega_{\mathrm{c}} n-\lambda\right)\left[\left(\omega_{\mathrm{c}} n-\lambda\right)^{2}-g^{2}(n+1)-g^{2} n\right]=0
$$

и, решая его, находим собственные векторы

$$
\begin{aligned}
|n, 0\rangle & =\sqrt{\frac{n+1}{2 n+1}}|1\rangle|n-1\rangle-\sqrt{\frac{n}{2 n+1}}|-1\rangle|n+1\rangle \\
|n, \pm\rangle & =\sqrt{\frac{n}{2(2 n+1)}}|1\rangle|n-1\rangle+\sqrt{\frac{n+1}{2(2 n+1)}}|-1\rangle|n+1\rangle \pm \frac{1}{\sqrt{2}}|0\rangle|n\rangle
\end{aligned}
$$

с соответствующими собственными значениями

$$
n \omega_{\mathrm{c}}, \quad n \omega_{\mathrm{c}} \pm 2 g \sqrt{n+\frac{1}{2}} .
$$

При $n=0$ мы имеем два уровня. Чтобы в этом убедиться, запишем соотношения

$$
\begin{aligned}
H_{0}|0,+\rangle & =\left(\omega_{\mathrm{c}} a^{+} a+\omega_{0} S_{z}+g\left(a^{+} S_{-}+a S_{+}\right)\right)(C|-1\rangle|1\rangle+B|0\rangle|0\rangle)= \\
& =\left(\omega_{\mathrm{c}}-\omega_{0}\right) C|-1\rangle|1\rangle+g C|0\rangle|0\rangle+B g|-1\rangle|1\rangle
\end{aligned}
$$

и $\left(\omega_{\mathrm{c}}-\omega_{0}\right) C+B g=\lambda C, g C=\lambda B$. Условие резонанса $\omega_{\mathrm{c}}-\omega_{0}=0$ дает $B g=\lambda C$, $g^{2}=\lambda^{2}, \lambda= \pm g$. Действие гамильтониана принимает вид

$$
\begin{aligned}
H_{0}|1,0\rangle & =\left(\omega_{\mathrm{c}} a^{+} a+\omega_{0} S_{z}+g\left(a^{+} S_{-}+a S_{+}\right)\right)\left(\sqrt{\frac{2}{3}}|1\rangle|0\rangle-\sqrt{\frac{1}{3}}|-1\rangle|2\rangle\right)= \\
& =\omega_{0} \sqrt{\frac{2}{3}}|1\rangle|0\rangle+g \sqrt{\frac{2}{3}}|1\rangle|0\rangle+g \sqrt{\frac{1}{2}}|0\rangle|0\rangle-\left(2 \omega_{\mathrm{c}}+\omega_{0}\right) \sqrt{\frac{1}{3}}|-1\rangle|2\rangle .
\end{aligned}
$$


8.2. Одевание. Запишем общую формулу для ПД (см. (30))

$$
\tilde{a}_{1}=a_{1}, \quad \tilde{a}_{0}=a_{0}+\left[a_{1}, s\right]-2(D s), \quad \text { где } \quad a_{1}=\frac{\sqrt{2} g}{\omega_{\mathrm{c}}}\left(S_{-}+S_{+}\right) .
$$

Рассмотрим инвариантное подпространство с заданным $n$. Удобно записать собственные векторы как матрицу

$$
\Phi=\left(\begin{array}{ccc}
\sqrt{\frac{n+1}{2 n+1}} \phi_{n-1} & \sqrt{\frac{n}{2(2 n+1)}} \phi_{n-1} & \sqrt{\frac{n}{2(2 n+1)}} \phi_{n-1} \\
0 & \frac{1}{\sqrt{2}} \phi_{n} & -\frac{1}{\sqrt{2}} \phi_{n} \\
\sqrt{\frac{n}{2 n+1}} \phi_{n+1} & \sqrt{\frac{n+1}{2(2 n+1)}} \phi_{n+1} & \sqrt{\frac{n+1}{2(2 n+1)}} \phi_{n+1}
\end{array}\right),
$$

так что $H \Phi=\Phi \Lambda$, где

$$
\Lambda=\operatorname{diag}\left(n \omega_{\mathrm{c}}+2 g \sqrt{n+\frac{1}{2}}, n \omega_{\mathrm{c}}, n \omega_{\mathrm{c}}-2 g \sqrt{n+\frac{1}{2}}\right) .
$$

Производная матрицы $\Phi$ имеет вид

$$
\Phi_{x}=\frac{a-a^{+}}{\sqrt{2}}\left(\begin{array}{ccc}
\sqrt{\frac{n+1}{(2 n+1)}} \phi_{n-1} & \sqrt{\frac{n}{2(2 n+1)}} \phi_{n-1} & \sqrt{\frac{n}{2(2 n+1)}} \phi_{n-1} \\
0 & \frac{1}{\sqrt{2}} \phi_{n} & -\frac{1}{\sqrt{2}} \phi_{n} \\
\sqrt{\frac{n}{(2 n+1)}} \phi_{n+1} & \sqrt{\frac{n+1}{2(2 n+1)}} \phi_{n+1} & \sqrt{\frac{n+1}{2(2 n+1)}} \phi_{n+1}
\end{array}\right),
$$

при этом

$$
\tilde{a}_{0}=a_{0}+\left[\frac{\sqrt{2} g}{\omega_{\mathrm{c}}}\left(S_{-}+S_{+}\right), s\right]-2 \frac{a-a^{+}}{\sqrt{2}} s .
$$

Используя (71), мы получаем множество интегрируемых потенциалов/моделей, возникающих из модели Дикке. Из функции $\phi_{0}=(1 / \sqrt{2 \pi}) e^{-x^{2} / 2}$ получается множество функций, порожденных действием повышающих и понижающих операторов. В данном базисе

$$
s=\operatorname{diag}\left(\frac{\sqrt{2}}{2} \frac{\phi_{1}}{\phi_{0}}, \frac{1}{\sqrt{2}} \frac{\phi_{0}}{\phi_{1}}-\frac{3}{2} \frac{\phi_{2}}{\phi_{1}}, \frac{\phi_{1}}{\phi_{2}}-\frac{\sqrt{3}}{\sqrt{2}} \frac{\phi_{3}}{\phi_{2}}\right) .
$$

Элементы данной матрицы в $x$-представлении задаются формулами

$$
\begin{array}{cl}
\frac{\phi_{1}}{\phi_{0}}=\sqrt{2} x, & \frac{\phi_{0}}{\phi_{1}}=\frac{1}{\sqrt{2} x}, \\
\frac{\phi_{2}}{\phi_{1}}=\frac{x^{2}+2 x-1}{x \sqrt{2}}, \quad \frac{\phi_{1}}{\phi_{2}}=\frac{x \sqrt{2}}{x^{2}+2 x-1}, \\
\frac{\phi_{3}}{\phi_{2}}=\frac{\sqrt{2}}{\sqrt{3}} \frac{x^{3}+2 x+1}{x^{2}-1+2 x} .
\end{array}
$$

ЗАмечАниЕ. В случае конечной размерности, учитывая равенство $H^{+}=H$, мы имеем, что обратная матрица для выбора нормировки есть $\Phi^{-1}=\Phi^{+}$, при этом $s=\Phi_{x} \Phi^{+}$. 
8.3. Цепочки ПД. Можно применить результаты п. 4.2, рассмотрев их наименее сложный вариант: один шаг итераций. Считая, что форма суперпотенциала фиксирована [5], запишем потенциал, имеющий инвариантную форму, для левой и правой цепочек соответственно как

$$
s_{n}^{+}=\eta_{n} a(x)+\xi_{n}, \quad s_{n}=\zeta_{n} b(x)+\rho_{n} .
$$

Подставим второе из этих равенств в формулу (37), в которой мы перегруппируем слагаемые и сделаем минимальное упрощение $D a_{1}=0$, получим

$$
a_{2} D\left(s_{n}+s_{n+1}\right)=a_{2}\left(s_{n}^{2}-s_{n+1}^{2}\right)-a_{1} s_{n+1}+s_{n} a_{1},
$$

это дает

$$
\begin{aligned}
& a_{2} D\left(\zeta_{n} b(x)+\rho_{n}+\zeta_{n+1} b(x)+\rho_{n+1}\right)= \\
& \quad=a_{2}\left(\left(\zeta_{n} b(x)+\rho_{n}\right)^{2}-\left(\zeta_{n+1} b(x)+\rho_{n+1}\right)^{2}\right)-a_{1}\left(\zeta_{n+1} b(x)+\rho_{n+1}\right)+\left(\zeta_{n} b(x)+\rho_{n}\right) a_{1} .
\end{aligned}
$$

Далее подставим первое равенство из (73) в формулу (29), записанную как

$$
\left(D s_{n}^{+}-D s_{n+1}^{+}\right) a_{2}=\left(s_{n+1}^{+}\right)^{2} a_{2}-\left(s_{n}^{+}\right)^{2} a_{2}-s_{n}^{+} a_{1}+\left[a_{1}, s_{n}^{+}\right]+\left(D a_{1}\right)+2 a_{2} D s_{n}^{+}+s_{n+1}^{+} a_{1},
$$

в результате получим условия на параметры $\zeta_{n}, \rho_{n}$, и функцию $b(x)$ :

$$
a_{2} D\left(s_{n}+s_{n+1}\right)=a_{2}\left(s_{n}^{2}-s_{n+1}^{2}\right)-a_{1} s_{n+1}+s_{n} a_{1}+D a_{1} .
$$

Аналогичные условия имеют место для $\eta_{n}, \xi_{n}$ и функции $a(x)$.

В случае гамильтониана Дикке (58) коэффициенты $a_{i}$ задаются с использованием выражений $(59)$ как $a_{2}=-\omega_{0} / 2, a_{1}=(g / \sqrt{2})\left(S_{-}+S_{+}\right)$. Наконец, для гамильтониана Дикке и его ближайших модификаций можно применить полином $b(x)=f x+g$ с потенциалом, заданным соотношением Миуры

$$
u_{n}=\frac{\omega_{0}}{2}\left(\zeta_{n} f+\left(\zeta_{n}(f x+g)+\rho_{n}\right)^{2}\right)-\frac{g}{\sqrt{2}}\left(S_{-}+S_{+}\right)\left(\zeta_{n}(f x+g)+\rho_{n}\right),
$$

выбирая подходящим образом значения коэффициентов.

\section{9. ЗАКЛЮЧЕНИЕ}

Бесконечномерное (операторное) одевание требует разработки некоммутативной техники. Например, можно исследовать такие вопросы, как задачи с рядами многократного рассеяния и сходимость соответствующего разложения [2]. Важным примером задачи с оператором более высокого порядка (по $D$ ) является также модель Хаббарда.

\section{Список литературы}

[1] V.B. Matveev, "Darboux transformations in associative rings and functional-difference equations", The Bispectral Problem (Montreal, PQ, March 1997), CRM Proceedings and Lecture Notes, 14, eds. J. Harnad, A. Kasman, AMS, Providence, RI, 1998, 211-226.

[2] E. V. Doktorov, S. B. Leble, A Dressing Method in Mathematical Physics, Mathematical Physics Studies, 28, Springer, Dordrecht, 2007. 
[3] Н. Н. Боголюбов, Н. Н. Боголюбов (мл.), Введение в квантовую статистическую механику, Наука, М., 1984.

[4] С. Б. Лебле, "Отклик классической многочастичной системы на двухимпульсное возбуждение”, Когерентное возбуждение конденсированных сред, ред. У. Х. Копвиллем, Дальневост. науч. центр АН СССР, Владивосток, 1979, 51-74; С. Б. Лебле, А. И. Иванов, Метод вторичного квантования, Калинингр. гос. ун-т, Калининград, 1981.

[5] С. Б. Лебле, "Метод одевания в квантовых моделях взаимодействия излучения с веществом", ТМФ, 152:1 (2007), 118-132.

[6] Л.И. Меньшиков, “Сверхизлучение и некоторые родственные явления”, УФН, 169:2 (1999), 113-154.

[7] Н. М. Боголюбов, П. П. Кулиш, Зап. науч. сем. ПОМИ, 398 (2012), 26-54.

[8] V.B. Matveev, "Darboux transformation and explicit solutions of the Kadomtcev-Petviaschvily equation, depending on functional parameters", Lett. Math. Phys., 3:3 (1979), $213-216$.

[9] S. Leble, A. Zaitsev, "Division of differential operators, intertwine relations and Darboux transformations", Rep. Math. Phys., 46:1-2 (2000), 165-174.

[10] P. Dubard, V. B. Matveev, "Multi-rogue waves solutions: from the NLS to the KP-I equation", Nonlinearity, 26:12 (2013), R93-R125. 